\title{
Rubber Additions into Concrete and Gamma Radiation Effects on Mechanical Properties and Microwave Absorption Capacity
}

\section{J COLÍN ${ }^{1}$, F. CASTILLO ${ }^{2}$, B. LEAL ${ }^{3}$, O FLORES ${ }^{2}$, I GAMBOA GLND H $^{3}$ MARTÍNEZ ${ }^{2}$}

\author{
${ }^{1}$ Facultad de Ciencias Químicas e Ingeniería, Universidad Autónoma del Estado de \\ Morelos, Av. Universidad 1001, Col. Chamilpa. C.P. 62209, Cuernavaca, Morelos, \\ México \\ ${ }^{2}$ Laboratorio de Espectroscopia, Instituto de Ciencias Físicas, Universidad Nacional \\ Autónoma de México, Apartado Postal 48-3, 62251, Cuernavaca, Morelos, México. \\ ${ }^{3}$ Instituto de Ciencias Nucleares, Universidad Nacional Autónoma de México, \\ Apartado Postal 70-543, 04510, Ciudad Universitaria México D.F. México
}

\section{Emial:}

Published online: August 07, 2017

The Author(s) 2017. This article is published with open access at www.chitkara.edu. in/publications

\begin{abstract}
Rubber is the indispensable raw material for the manufacture of tires; it is obtained from plants, trees and currently can be produced synthetically. The tire rubber is mixed with compounds such as carbon black, sulfur, cement, paints, antioxidants, oils and fats, steel wire, almost etc., making impossible to recycle the tires itself. In this work, we investigate, the effects of the incorporation of ground rubber in concrete, mixture designed to establish the possibility of being used in the construction industry. The samples of concrete were addition with, 5 vol. \%,, 15 vol. $\%$ and 25 vol. $\%$ of rubber. Samples with different rubber addition were irradiated by a cobalt 60 gamma source to study the effect on their mechanical compression properties and microwave absorption capacity. Likewise, the microwave absorption capacity was studied for both irradiated and the non-irradiated. It was found that between 5 vol. $\%$ and 15 vol. $\%$ of rubber addition change the mechanical properties approximately $25 \%$, higher rubber additions result in a decrease in a $75 \%$ of its mechanical properties. The fracture behavior is not the expected one due to as the curves of stress vs. strain show a double slope, which is associated with the concrete porosity and rubber content. The aging with the gamma rays generates loss of mechanical properties, especially at lower rubber addition, since at content greater than $15 \mathrm{Vol} \%$ the radiation effect is less. These rubber
\end{abstract}

Journal of Nuclear

Physics, Material Sciences, Radiation and Applications Vol-5, No-1, August 2017 pp. 197-211 
Colín, J

Castillo, F

Lea, B

Flores, O

Gamboa, I

Martínez, H additions allow moderate deformations in compression, thus contributing to the care and preservation of the environment.

Keywords: Concrete, Waste tire rubber, Microwave absorption, Gamma radiation, Mechanical properties, Ecological materials.

\section{INTRODUCTION}

The construction industry is an activity that greatly influences the economic development of the countries but at the same time generates great impact on the environment by being in continuous interaction with it, starting from the obtaining of raw materials up to the alteration of the earth's crust. The industry concrete is the preponderant raw material that has evolved according to the needs of the builders, facing properties such as strength, durability, work or aesthetics, bringing with it, a wide variety of alternatives that bring efficiency and innovation. On the other hand, in the last years has increased the world automotive fleet which has caused the increase of tire use and once the useful life of these tires has become a great environmental problem due to the difficulty of their reuse. The tires are basically a rubber cover which must be able to support the weight of the vehicle and its load, according to the design and use. Different materials such as textiles, rubber and metals are used for their manufacture. Concrete is a mixture of various elements that properly dosed provide properties according to the needs of the work to be performed and the regulations and specifications of the countries where it is manufactured. Among the elements that compose it are the aggregates, water, air, and cement, where each plays a fundamental role in the mixture. The present work focuses on the reduction of negative effects on the environment, recovering waste for alternative use in raw materials of the concrete and automotive industry. What sought is to evaluate the possibility of designing a concrete with aggregates recycled material of ground rim.

The mixing design of a concrete is mainly based on achieving a certain compressive strength at a specific age and complying with the properties that the concrete must have, when the structure is in service. The design mixtures will be made using the Graphical method. This method allows finding more appropriately the dosage of the coarse and fine aggregates including those whose granulometry does not comply with the limits established in the standard because it optimizes the gradation reaching the maximum density. Simple compressive strength is the main mechanical characteristic of concrete. The way to express it is in $\mathrm{kg} / \mathrm{cm}^{2}$. The best way to evaluate the concrete is by means of mechanical tests for which the established norm is used. The present research project aims 
to develop different types of low cost concrete, added with millimeter particles and micrometric rubber for specific applications requiring the ability to undergo moderate elastic deformations without breaking, thermal and acoustic insulation, damping of mechanical vibrations And resistance to the formation of catastrophic cracks. Based on the above, it is proposed to develop elastic deformable cement + sand + rubber (concrete) composites with modified mechanical and acoustic properties by introducing rubber particles from recycled tires.

Concrete is the mixture of coarse and fine aggregates with a cementing paste composed of Portland cement and water, which formed monolithic mass similar to rock. The paste hardens due to the chemical reaction between water and Portland cement, generating hydration products.

The aggregates are natural or synthetic sands with maximum sizes up to 10 $\mathrm{mm}$ and coarse aggregates are rocks or gravels with maximum sizes up to 152 $\mathrm{mm}$. Both aggregates consist of between 60 and $75 \%$ of the total volume of the mixture; therefore they can limit the properties of the concrete and influence its performance. However, these properties depend largely on the quality of the paste formed by water and cement, as well as the affinity that they have with aggregates and their ability to work together.

A fundamental and perhaps most important physical property of concrete in a hardened state is its compressive strength (maximum load that it can withstand per unit area). This index is used in the calculation, design and elaboration of the mixtures that will be used to make elements that will be part of the structures.

Among the main factors that determining their resistance are: concrete age, water-cement ratio of the mixture, degree of compaction of aggregates in concrete, porosity and air content trapped at the time of manufacture concrete. Other variables involved in the calculation of resistance are aggregate-cement ratio, aggregate quality (grain size, surface texture, shape, strength, stiffness and nominal size).

Portland cement is obtained from the mixture of calcareous materials (limestone or gypsum), silica, alumina and iron oxide. The main chemical components of Portland cement are $3 \mathrm{CaO}-\mathrm{SiO}_{2}\left(\mathrm{C}_{3} \mathrm{~S}\right)$ tricalcium silicate, dicalcium silicate $2 \mathrm{CaO}-\mathrm{SiO}_{2}\left(\mathrm{C}_{2} \mathrm{~S}\right)$, Tricalcium aluminum $3 \mathrm{CaO}-\mathrm{Al}_{2} \mathrm{O}_{3}\left(\mathrm{C}_{3} \mathrm{~A}\right)$ and Aluminoferrite $4 \mathrm{CaO}-\mathrm{Al}_{2} \mathrm{O}_{3} \cdot \mathrm{Fe}_{2} \mathrm{O}_{3}\left(\mathrm{C}_{3} \mathrm{~S}\right)$ (Neville \& Brooks, 1998 ).

In concrete, an alternative that has proven to be efficient to increase resistance values (1), it is the use of gamma radiation through two methods:

- Modification of the structural properties of reinforcements and their subsequent incorporation into concrete mixtures.

- Direct irradiation of concrete with reinforcements included.
Rubber Additions into Concrete and Gamma

Radiation Effects on Mechanical Properties and Microwave Absorption Capacity 
Colín, J

Castillo, F

Lea, B

Flores, O

Gamboa, I

Martínez, H

So, based on the above described, the objective of the present work is to develop concrete matrix composites modified with waste tire rubber to obtain elastically deformable materials in addition to enhanced microwave absortion properties.

\section{EXPERIMENTAL SETUP}

Four concrete blocks, three of which were mixed with rubber scratches of waste recycle tires, were prepared according to the compositions shown in Table 1. The cement used in the concrete was of the Portland CPC 30 type.

The rubber used was of the type "Scratched" that was obtained from scrap tires, figure 1.

Once the mixtures were made, they were emptied in wood molds oiled on the walls, to form continuous bars of square section of $5 \mathrm{~cm}$ of side by $30 \mathrm{~cm}$ of length, figure 2 .

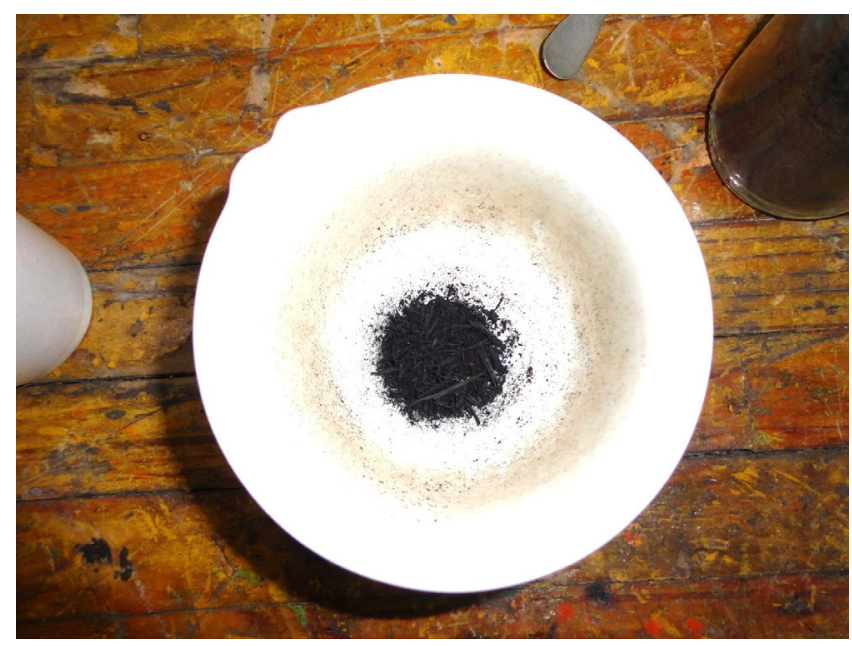

Figure 1: Scrap tire rubber scratches obtained by mechanical milling.

Table 1: Proportions of concrete + rubber used in the samples under study.

\begin{tabular}{ccc}
\hline Sample & Rubber content (Vol. \%) & Concrete (Vol. \%) \\
\hline M1 & 0 & 100 \\
M2 & 5 & 95 \\
M3 & 15 & 85 \\
M4 & 25 & 75 \\
\hline
\end{tabular}



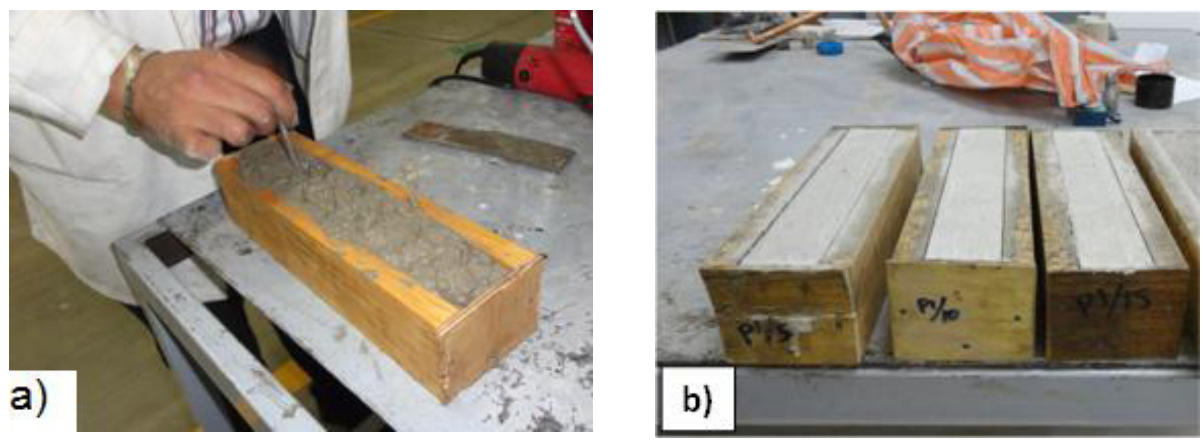

Rubber Additions into Concrete and Gamma

Radiation Effects on Mechanical Properties and Microwave Absorption Capacity

Figure 2: a) The mixtures were cast and degasified in wood molds. B) Appearance of bars after 24 hours after casting.

Subsequently, each bar of specific composition was sectioned to obtain cubes of $75 \mathrm{~cm}^{3}$ and square slices $1 \mathrm{~cm}$ thick.

When a polymeric material is subjected to ionizing radiation, physical and chemical effects are produced depending on the nature of the polymers as well as the dose of the radiation. The advantage of working with this type of macromolecules is the great sensitivity to changes in chemical bonds, obtaining different properties in terms of crystalline, density, coefficient of thermal expansion, modulus of elasticity, permeability, as well as corrosion resistance, Abrasion and solvents.

The number of cube and slice samples of each composition was divided into two equal parts to form groups of which one was subjected to $35 \mathrm{kGy}$ doses of gamma radiation of cobalt 60 using a radiator Gammabeam 651 PT variable dose rate with $90 \mathrm{kCi}$, the concrete samples were irradiated at a dose rate of $10 \mathrm{kGy} / \mathrm{hr}$ at a dose of $35 \mathrm{kGy}$. The obtained samples, both the Radiated (R) and the No Radiated (SR) were characterized by compression tests in an Instron universal machine for mechanical tests, model 4206 of 150 $\mathrm{kN}$ of capacity, using a speed of deformation of $0.5 \mathrm{~mm}$ per minute.

After irradiation, the structural changes of the reinforcements are related to the mechanical properties of the concrete, including tensile strength, compression; impact, as well as deformation at the point of yield and rupture. There is little research to evaluate the effect of gamma radiation on concrete.

The calculation of the modulus of elasticity of the concrete is a complex subject since there are different criteria and proposals to calculate it $[3,4,5]$, however, in the present work was calculated by the equation 1 according to the established criterion By the American Concrete Institute (ACI).

$$
E_{0}=4700 \sqrt{f_{c}^{\prime}}
$$


Colín, J

Castillo, F

Lea, B

Flores, O

Gamboa, I

Martínez, $\mathrm{H}$

where:

$$
\begin{aligned}
& E_{0}=\text { Modulus of Elasticity (Mpa) } \\
& f^{\prime}{ }_{c}=\text { Stress Strength of concrete (Mpa) }
\end{aligned}
$$

In the other hand, the stress strength was determined using a strain criterion of $0.01 \%$.

In order to know if this new concrete mix serves to isolate some of the electromagnetic signals present in the environment, studies of microwave penetration were carried out on the different samples. To do this we use a microwave guide, consisting of a T-shaped guide, two square waveguides, a klystron microwave generator JAN-CRP 2K25 and at the opposite end, an HP $\mathrm{X}-424 \mathrm{~A}$ PIN diode detector for the detection of the microwave signal.

\section{RESULTS}

\subsection{Mechanical properties}

The stress vs. strain curves of the R and SR samples tested at different compositions, showed a similar behavior between them (see figure 3), it can observe the presence of two slopes in all samples. The first corresponds to the "elastic" behavior of the material in which the linearity of the deformation with respect to the stress is interrupted by the appearance of a cracking, see figure 4 , showing a maximum value followed by a small drop. Then, the stress value increases again as does the deformation too, forming the second slope that exhibits a "semi-linear" behavior corresponding to the "quasi-elastic" behavior of the cracked material (see figure 3).

a)

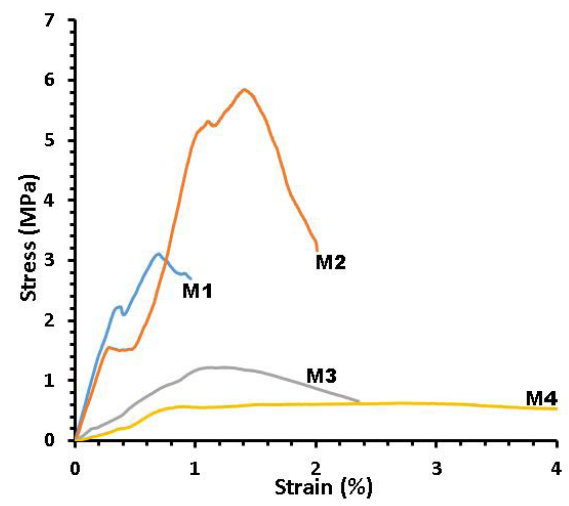

b)

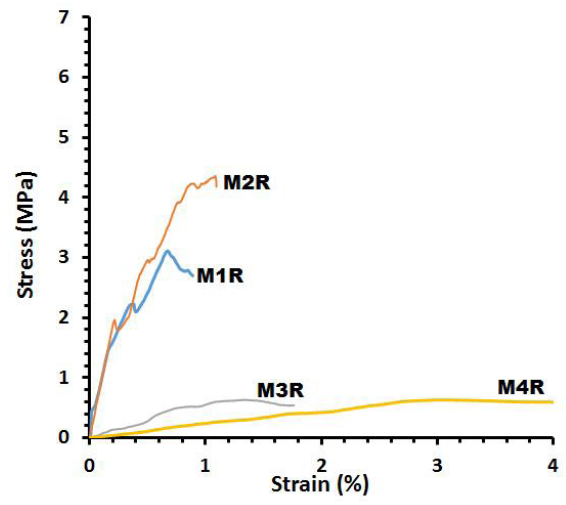

Figure 3: Stress vs. Strain curves obtained in the samples: a) No Radiated; b) Radiated. 


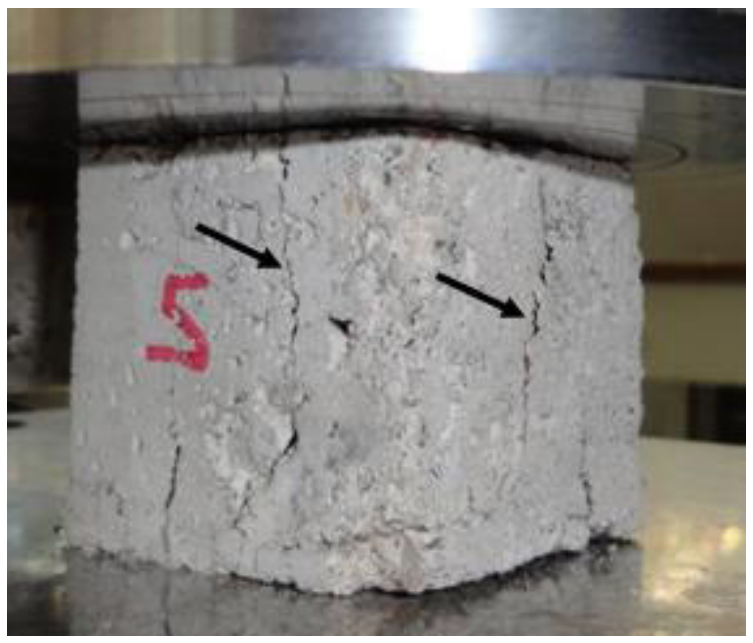

Rubber Additions into Concrete and Gamma

Radiation Effects on Mechanical Properties and Microwave Absorption Capacity

Figure 4: Representative photograph of the cracking observed during the compressive test of the samples with their respective compositions.

The stress value observed when the crack appears, corresponds to the stress strength of the material and it was determined from the graphs of figure 3 using the $0.01 \%$ criterion described in the experimental procedure. As expected, sample M1 corresponding to the sample without rubber additions, which exhibits the highest stress strength of $3 \mathrm{MPa}$, followed by sample M2 with rubber content of $5 \mathrm{Vol} \%$ and stress strength of $1.86 \mathrm{MPa}$. After that, the stress strength drastically decreases to $1.54 \mathrm{Mpa}$ and $0.52 \mathrm{Mpa}$ values corresponding to the M3 and M4 samples respectively.

Similar behavior was observed in the $\mathrm{R}$ samples, noting that the higher values of stress strength correspond to the M1R with no rubber additions and M2R with 5 Vol \% of rubbers addition.

When comparing the mechanical properties of the samples M1 and M2 with M1R and M2R (figure 5), it can observed that the radiation, apparently, it has an adverse effect on the mechanical resistance of the pure concrete, since the stress strength value of the concrete sample without radiation (M1) is higher than that of the radiated concrete sample (M1R).

On the other hand, an apparently beneficial of the radiation effect on the modified sample with $5 \mathrm{Vol} \%$ rubbers addition is observed since the stress strength showed by the M2R sample is higher than the exhibited by the M2 sample (see figure 5).

An important point to note is that the modification of the concrete with rubber scratches produces changes on its deformation capacity as it can be seen 
Colín, J

Castillo, F

Lea, B

Flores, $\mathrm{O}$

Gamboa, I

Martínez, H

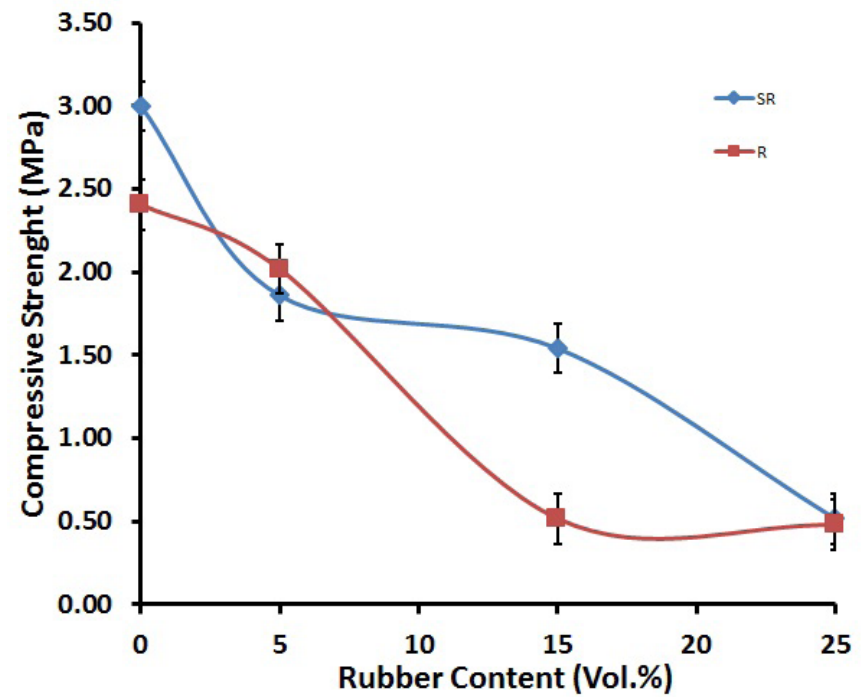

Figure 5: Stress strength variation as a function of the rubber content in the radiated (R) and no radiated (SR) samples.

in figure 6. It can be appreciated that non radiated samples (SR) exhibits an oscillatory behavior of the deformation at its different compositions, however, an increase tendency in the deformation as a function of the rubber content in the sample can be assumed.

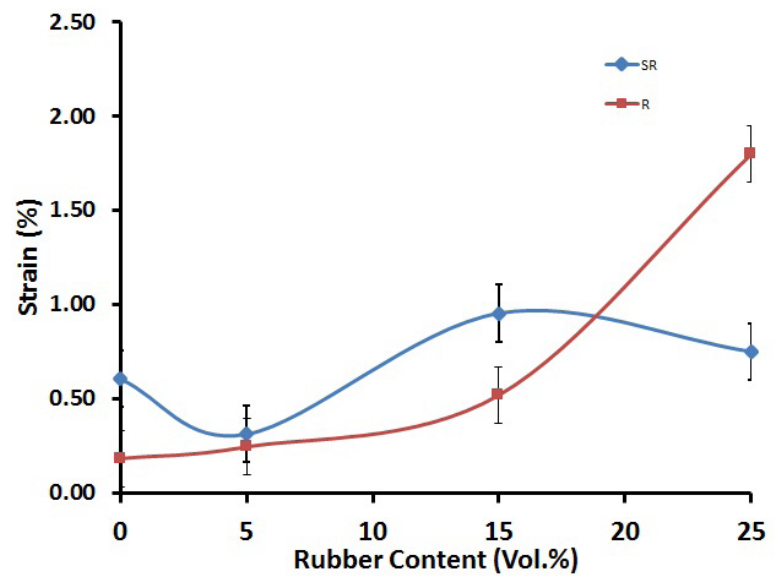

Figure 6: Maximum strain (deformation) registered in the samples, previously to their cracking. 
Otherwise, the deformation of the radiated samples shows a regular behavior respecting the rubber content observing a uniform increase in the $5 \mathrm{Vol} . \%$ (M2R) and $15 \mathrm{Vol} . \%$ (M3R) samples, which respectively registered $0.25 \%$ and $0.52 \%$ as deformation values. The maximum deformation value was of $1.8 \%$ and corresponds to the M4R sample. Table 2 resumes the results of the mechanical tests.

\subsection{Structural Characterization}

The samples used in the compressive test were macroscopically observed during and after the test performance in order to determine the sample behavior during the load and the fracture type as well as the distribution of the rubber scratches in the already fractured samples.

It was observed that in the samples without rubber additions (M1 and M1R), when they cracking tend to disintegrate, noting that the crack rapidly advances and immediately separated the material and the sample collapsed (Figure 7). On the other hand, in the samples with $5 \mathrm{Vol} \%$ of rubber addition and later, figure 8 a) and b), the behavior of the cracking was different observing that the formed crack advanced slowly, without a sudden separation of the material that would lead to its disintegration.

When observing the fractures of the samples at different compositions, it was found that, in those that do not contain rubber scratches, internal cracks mainly wide are formed and propagated through the matrix of cement and fine aggregate (sand) and even through the coarse aggregate (gravel) (see figure 9 a)), separating them completely and giving rise to the disintegration and collapse of the material.

The opposite happens in rubber-modified samples where narrow cracks are observed which do not seem to evolve or widening or even growing fast (see figure 9 (b)). This behavior is presumably due to the fact that rubber stripes,

Table 2: Numerical results of the mechanical tests performed on the R and SR samples at its different rubber contents.

\begin{tabular}{ccccccc}
\hline \multirow{2}{*}{ Sample } & \multicolumn{2}{c}{$\begin{array}{c}\text { Compressive } \\
\text { Strength (Mpa) }\end{array}$} & \multicolumn{2}{c}{ Strain (\%) } & \multicolumn{2}{c}{ Modulus of Elasticity } \\
\cline { 2 - 7 } & SR & R & SR & R & $E_{0}$ & $E_{0 R}$ \\
\hline M1 & 3.00 & 2.41 & 0.61 & 0.18 & 8141.32 & 7293.33 \\
M2 & 1.86 & 2.02 & 0.31 & 0.25 & 6409.34 & 6679.74 \\
M3 & 1.54 & 0.52 & 0.95 & 0.52 & 5836.00 & 3381.77 \\
M4 & 0.52 & 0.48 & 0.75 & 1.80 & 3377.25 & 3253.00 \\
\hline
\end{tabular}

Rubber Additions into Concrete and Gamma

Radiation Effects on Mechanical Properties and Microwave Absorption Capacity 
Colín, J

Castillo, F

Lea, B

Flores, O

Gamboa, I

Martínez, H

seem to be "anchors" (see figure 10 a)), which, although they are not capable of preventing cracking of the material due to its intrinsic fragility, apparently they are capable of inhibiting the rapid advance of the crack and even the blockage of the crack to a certain limit that is determined by the intensity of the load supported. Basically the rubber striped avoids the abrupt separation of the fractured parts and therefore, the disintegration and collapse of the material (see figure 10).

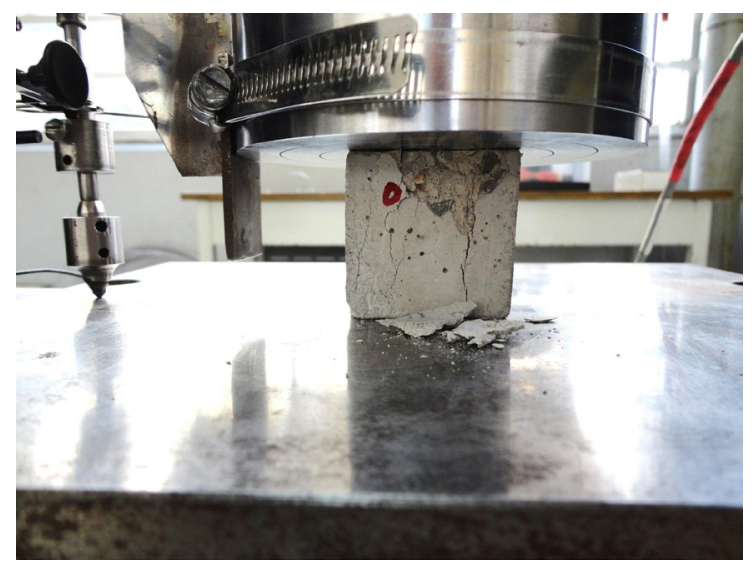

Figure 7: Representative photograph of the mechanical behavior of the samples without rubber additions in the $\mathrm{R}$ and $\mathrm{SR}$ conditions. It can be seen that during the compression test, the material tends to disintegrate once the cracking has started.
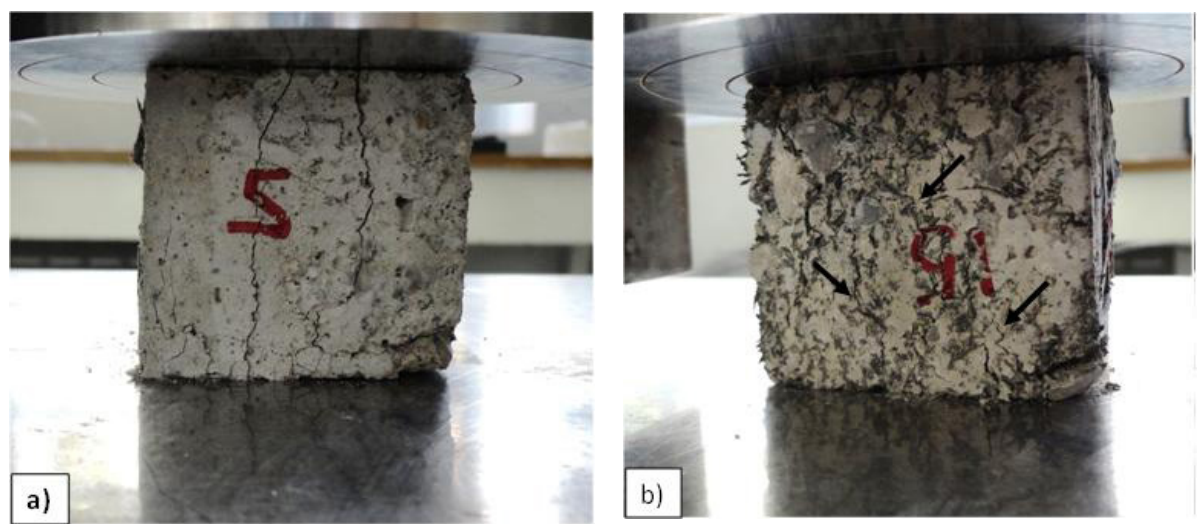

Figure 8: Distinctive photograph of the mechanical behavior of SR and R samples with: a) $5 \mathrm{Vol} \%$ rubber; b) $15 \mathrm{Vol} \%$ rubber during the compressive test. Note that the sample cracks but does not disintegrate as in samples with no rubber additions. 

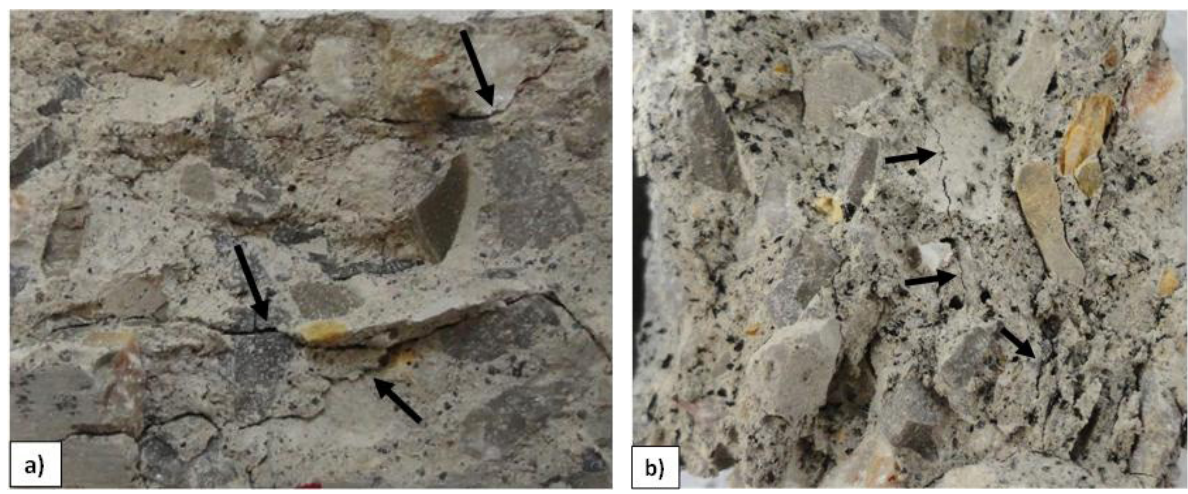

Rubber Additions into Concrete and Gamma

Radiation Effects on Mechanical Properties and Microwave Absorption Capacity

Figure 9: Macrofotographs of fractures observed in a) samples without rubber; b) with rubber scratches.
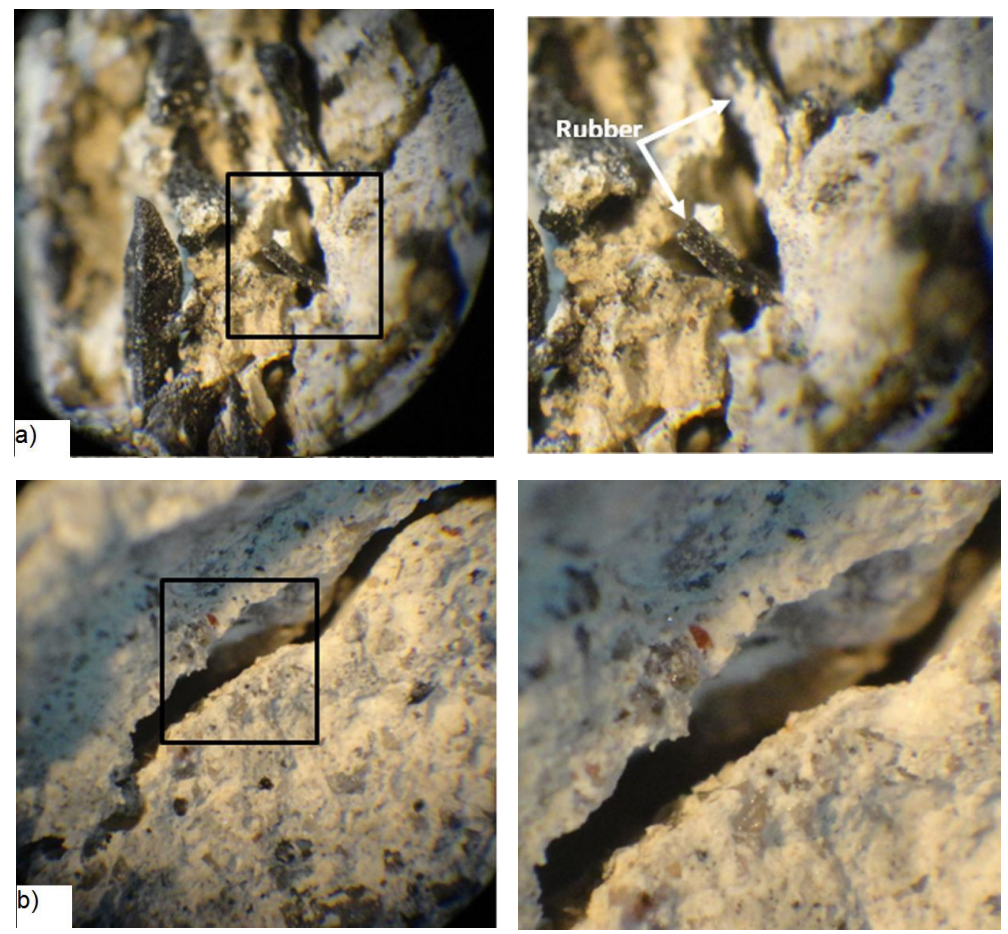

Figure 10: Micrographs of fractures in a) sample with rubber; B) sample without rubber. The images on the right are amplifications of the areas represented by the boxes. 
Colín, J

Castillo, F

Lea, B

Flores, O

Gamboa, I

Martínez, $\mathrm{H}$

\subsection{Microwaves}

The term microwave includes electromagnetic radiation included in the frequency band extending from $300 \mathrm{MHz}$ to 3,000 GHz. In its interaction with matter, its energy can be reflected, as it happens when it hits metallic surfaces, transmitted with little loss of energy in transmitting media, such as glass, or absorbed by the irradiated matter, which causes an increase in temperature in this.

The attenuation of the microwaves obeys to the exponential law of Lambert-Beer:

$$
\mathrm{I}=\mathrm{I}_{\mathrm{o}} \times \mathrm{e}^{-\alpha x}
$$

Where:

$I_{0}$ : incident intensity

I: Output current after passing through a given material

$\mathrm{x}$ : thickness of the material traversed

$\alpha$ : attenuation coefficient

The attenuation coefficient $(\alpha)$ is a characteristic of each medium. If this coefficient is very large, the medium absorbs a lot and will decrease the penetration. Therefore, the attenuation coefficient and the depth of penetration are in inverse ratio. This coefficient also depends on the wavelength and the nature of the medium.

The penetration results to be proportional to the wavelength, since, with smaller wavelengths, the attenuation coefficient increases. If the medium is rich in water, the absorption at its level is greater. Hence, the dielectric permittivity, magnetic permeability and electrical conductivity of the medium also influence.

The samples (the three added with rubber and the normal) both the radiated and the non-irradiated were placed in a rectangular waveguide system, to study the passage of the microwaves through the samples, and to see the effect.

The effects of this are shown in Figure 11, where the attenuation of the microwaves is observed, for the first two samples added with rubber. It is observed that for both radiated and non-irradiated samples, the attenuation is higher for the sample with a 5\% addition of rubber. It can also be observed that the sample with $25 \%$ rubber addition, in both cases, behaves as the sample without rubber.

In conclusion, we see that having more than $5 \%$ of added rubber, is not a benefit to attenuate or stop the passage of the microwaves of this frequency by the samples. We see that the radiated samples attenuate more the microwaves, when they are added with $5 \%$ of rubber. The latter is not very clear why. 


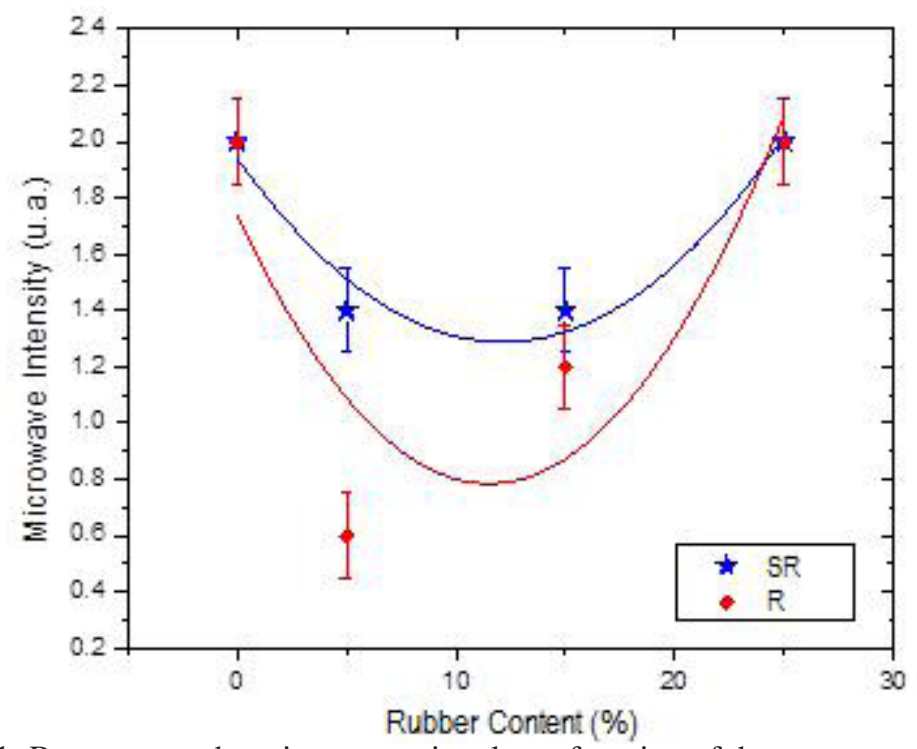

Rubber Additions into Concrete and Gamma

Radiation Effects on Mechanical Properties and Microwave Absorption Capacity rubber.

\section{CONCLUSIONS}

The results obtained in the present study show a decrease in the mechanical properties of the concrete when modified with rubber addition, which is in agreement with the reported in the literature [6, 7, and 8]. However, it has been reported that such a decrease can range from $50 \%$ to $85 \%$ [7, 9, and 10] as a function of the rubber content. The most notable decrease in stress strength was $50 \%$ which was obtained by Felipe J.A. And Jeannette Santos [10] with a concrete mix with $14 \%$ rubber. In the present work, a reduction of $48 \%$ of the stress strength was obtained with a rubber content of $15 \%$ vol. It is not possible to adequately compare both results since it is unknown whether the \% rubber specified by Felipe J.A. And Jeannette Santos [10] is by volume or by weight.

The most remarkable results of the present work are: a reduction of $38 \%$ of stress strength was obtained in the non-radiated (SR) sample with 5 Vol. \% of rubber and $32 \%$ in the sample with the same rubber content but radiated (R).

It is presumed that the gamma radiation has a beneficial effect on the compressive strength of the modified sample with $5 \mathrm{Vol}$. \% of rubber since it exhibited a greater value of stress strength than the one observed in the sample with the same composition but without radiation

Samples without rubber additions, both $\mathrm{R}$ and SR, exhibit a fragile behavior since it was observed that the formed cracks are wide and fragments the material, producing a crumbling and its fast collapse. 
Colín, J

Castillo, F

Lea, B

Flores, O

Gamboa, I

Martínez, H

Rubber-modified samples, both $\mathrm{R}$ and SR, show a slow cracking and a smooth crack advance, without completely shattering or crumbling. This is presumably because the rubber scratches, due to its morphology like elongated fibers, keep the fragments together, preventing their separation and collapse of the material

Microwave absorption tests results suggest that, there is a beneficial effect of the gamma radiation on the microwave absorption capacity of the samples being such effect remarkable in the sample M2R with a rubber content equal 5 vol \%.

These results open the possibility of investigations with great benefits, on the one hand the use of recycled scrap tires in concrete and on the other, the improvement of the properties of the same. It is desired to ensure the economic benefit in the context of sustainable development, which involves solving the problem of environmental pollution caused by waste tires, to achieve the welfare of the population improving their quality of life.

\section{ACKNOWLEDGEMENT}

The authors are thankful to H.H. Hinojosa for their technical assistance. DGAPA IN-102916. To A. Aguilar Negrete, J. Macedonio Andrés and L. Brito Rodríguez from TAMULBA, FCQeI-UAEM for their technical support. To the Convenio de Colaboración UNAM-UAEM No. 42467-2177-8-IX-15.

\section{REFERENCES}

[1] Acosta F. J., Santos J., Suarez O. M. and Pando M.A. (2007) "Raising Awareness on Materials Recycling using Undergraduate Engineering Research," International Journal of Environment and Pollution, Vol. 31, No. 3/4, pp. 325-341.

[2] Domingo J. Carreira and Kuang-Han Chu, Stress-Strain Relationship for Plain Concrete in Compression, ACI JOURNAL I November-December 1985.

[3] Del Villar Alvelais E., Saneamiento de sitios contaminados con llantas de desecho y gestión de residuos de llantas de desecho, conferencia de legisladores fronterizos. Tampico, tamps., 6 DE MARZO DE 2009

[4] Eldin, N.N., Senouci, A.B., (1993). Rubber-tire particles as concrete aggregates. ASCE, Journal of Materials in Civil Engineering 5 (4), 478-496.

[5] ISHTIAQ A., UMER A., NOUMAN K., Use of Rubber as Aggregate in Concrete: A Review, International Journal of Advanced Structures and Geotechnical Engineering. ISSN 2319-5347, Vol. 04, No. 02, April 2015

[6] Flores L., El Economista (México), 2 de mayo de 2016 (37) 
[7] N. Oikonomou* and S. Mavridou, RUBCRETE - RUBBERIZED PORTLAND CEMENT CONCRETE, In: Progress in Sustainable Development Research, pp. 201-214. Nova Science Publishers, Inc. ISBN: 978-1-60021-847-7.

[8] Siddique R., R. Tarun Naik, Properties of concrete containing scrap-tire rubber an overview, Waste Management 24 (2004) 563-569.

[9] Standard Method of Static Test of Young's Modulus of Elasticity on Poissons Ratio in compression of Cylindrical Concrete Specimens (ASTM C 469-65). Filadelfia, USA, American Society for Testing and Materials, 1965.

[10] T. Noguchi, F. Tomosawa, Kamran M. Nemati, Bernardino M. Chiaia, and Alessandro P. Fantilli, A Practical Equation for Elastic Modulus of Concrete, ACI Structural Journal/September-October 2009.
Rubber Additions into Concrete and Gamma
Radiation Effects on Mechanical Properties and Microwave Absorption Capacity

